\title{
Meson Spectrum, Glueball Mass and QCD Effective Coupling within Infrared Confinement
}

\section{Gurjav Ganbold*}

Bogoliubov Laboratory of Theoretical Physics, JINR, 141980, Dubna, Russia

Institute of Physics and Technology, 210651, Ulaanbaatar, Mongolia

E-mail: ganboldetheor.jinr.ru

Bound states of spin-half and spin-one particles are studied within a relativistic quantum-field model based on the infrared confinement. The spectra of quark-antiquark and two-gluon states are determined by master equations similar to the ladder Bethe-Salpeter equations. Masses of light, intermediate and heavy mesons are estimated in a wide range of scale (up to $10 \mathrm{GeV}$ ) and a new analytic expression is obtained for the lowest-state glueball mass. A new, independent and specific infrared-finite behavior of QCD effective coupling is revealed in the low-energy domain.

Xth Quark Confinement and the Hadron Spectrum,

October 8-12, 2012

TUM Campus Garching, Munich, Germany

\footnotetext{
* Speaker.
} 


\section{Introduction}

Understanding of a number of phenomena such as quark confinement, hadronization and nonvanishing vacuum expectation values, etc. requires a correct description of hadron dynamics in the infrared (IR) region below $Q \sim 1 \mathrm{GeV}$ [四]. Particularly, many quantities in particle physics are affected by the IR behavior of the QCD effective coupling $\alpha_{s}$. QCD predicts a dependence of the physical coupling $g$ under changes of distance $\sim 1 / Q$. This dependence $\alpha_{s}(Q) \doteq g^{2} /(4 \pi)$ is described theoretically by the renormalization group equations and determined experimentally at relatively high energies [2]. Nevertheless, the long-distance behavior of $\alpha_{s}$ is not well defined, it

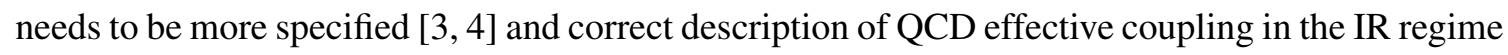
remains one of the actual problems in particle physics. It represents a certain interest to investigate some low-energy physics problems, such as hadronisation, glueball states, QCD effective (running) charge within a simple relativistic model based on physically transparent hypotheses, which can be treated by simple analytic methods.

Below, we take into account the dependence of $\alpha_{s}$ on mass scale $M$ and determine the QCD effective charge in the low-energy region by exploiting the hadron spectrum [[5].

\section{Model with IR-confined Propagators}

Recent theoretical results predict an IR-finite behavior of the gluon propagator [ [6, 团]. We follow this conception and consider the gluon and quark propagators exhibiting explicit IR-finite behaviors as follows:

$$
\tilde{D}(p)=\frac{1}{p^{2}}\left(1-e^{-p^{2} / \Lambda^{2}}\right), \quad \tilde{S}_{m}(\hat{p})=\frac{i \hat{p}+m_{f}}{p^{2}+m_{f}^{2}}\left(1-e^{-\left(p^{2}+m_{f}^{2}\right) / \Lambda^{2}}\right),
$$

where $\Lambda$ is the IR confinement scale. These propagators are entire analytic functions in the Euclidean space. The confinement disappears as $\Lambda \rightarrow 0$.

For the spectra of two-quark bound states we develop a relativistic quantum-field model based on IR confinement and consider the model Lagrangian [Q]:

$$
\mathscr{L}=-\frac{1}{4}\left(F_{\mu v}^{A}\right)^{2}+\left(\bar{q}_{f}^{a}\left[\gamma_{\alpha} \partial^{\alpha}-m_{f}\right]^{a b} q_{f}^{b}\right)+g\left(\bar{q}_{f}^{a}\left[\Gamma_{C}^{\alpha} \mathscr{A}_{\alpha}^{C}\right]^{a b} q_{f}^{b}\right),
$$

where $\mathscr{A}_{\alpha}^{C}$ is the gluon field, $F_{\mu v}^{A}=\partial^{\mu} \mathscr{A}_{v}^{A}-\partial^{v} \mathscr{A}_{\mu}^{A}-g f^{A B C} \mathscr{A}_{\mu}^{B} \mathscr{A}_{v}^{C}, q_{f}^{a}$ is a quark field of flavor $f$, and $\Gamma_{C}^{\alpha}=i \gamma_{\alpha} t^{C}$. The model parameters are $\Lambda$ and the constituent quark masses $m_{f}=$ $\left\{m_{u d}, m_{s}, m_{c}, m_{b}\right\}$.

By omitting intermediate stages of calculation (see for details [ [8, [0]]) we rewrite the master equation determining the meson masses as follows:

$$
\begin{aligned}
& 1+\alpha_{s} \cdot \lambda_{J}\left(M_{J}^{2}\right)=1+\frac{16 \pi \alpha_{s} C_{J}}{9} \iint d x d y U_{N}(x) \sqrt{D(x) D(y)} U_{N}(y) \\
& \cdot \int \frac{d^{4} k}{(2 \pi)^{4}} e^{-i k(x-y)} \operatorname{Tr}\left[O_{J} \tilde{S}_{m_{1}}\left(\hat{k}+\xi_{1} \hat{p}\right) O_{J} \tilde{S}_{m_{2}}\left(\hat{k}-\xi_{2} \hat{p}\right)\right]=0, \quad p^{2}=-M_{J}^{2},
\end{aligned}
$$

where $C_{J}=\{1,1,1 / 2,-1 / 2\}, \xi_{i}=m_{i} /\left(m_{1}+m_{2}\right), O_{J}=\left\{I, i \gamma_{5}, i \gamma_{\mu}, \gamma_{5} \gamma_{\mu}\right\}$ and the polarization kernel $\lambda_{J J^{\prime}}\left(-p^{2}\right)$ has been diagonalized on a complete system of orthonormal functions $\left\{U_{N}\right\}$. Note, 
solution of Eq.([2.3]) is nothing else but the solution of the corresponding ladder Bethe-Salpeter equation.

The dependence of meson masses on $\alpha_{s}$ and other model parameters is defined by Eq.(R.3)). Note, the kernel function $\lambda_{N}$ is real and finite, it allows us to derive both analytic and numeric solutions.

1) An asymptotic Regge-type behavior is observed: $M_{J}^{2} \approx M_{0}^{2}+J \cdot c(M)$ for $J \geq 3$.

2) For the same quark-antiquark content a vector meson is heavier than its pseudoscalar counterpart: $M_{V}>M_{P}$ because of $C_{V}<C_{P}$.

3) We derive meson mass formula Eq.([2.3]) and adjust the model parameters by fitting heavy meson masses $(M \geq 2 \mathrm{GeV})$. Particularly, we fix a set of model parameters (in units of $\mathrm{MeV}$ ):

$$
\Lambda=220, m_{u d}=247.2, m_{s}=432.5, m_{c}=1544.5, m_{b}=4740.9 .
$$

4) As application we calculate intermediate and heavy meson masses shown in Table 1.

\begin{tabular}{|c|c||c|c||}
\hline$J^{P C}=0^{-+}$ & $M$ & $J^{P C}=1^{--}$ & $M$ \\
\hline$D(1870)$ & $1892 \mathrm{MeV}$ & $\rho(770)$ & $771 \mathrm{MeV}$ \\
$D_{s}(1970)$ & $1998 \mathrm{MeV}$ & $K^{*}(892)$ & $893 \mathrm{MeV}$ \\
$\eta_{c}(2980)$ & $3042 \mathrm{MeV}$ & $D^{*}(2010)$ & $1961 \mathrm{MeV}$ \\
$B(5279)$ & $5117 \mathrm{MeV}$ & $D_{s}^{*}(2112)$ & $2079 \mathrm{MeV}$ \\
$B_{s}(5370)$ & $5232 \mathrm{MeV}$ & $J / \Psi(3097)$ & $3097 \mathrm{MeV}$ \\
$B_{c}(6286)$ & $6238 \mathrm{MeV}$ & $B^{*}(5325)$ & $5168 \mathrm{MeV}$ \\
$\eta_{b}(9389)$ & $9384 \mathrm{MeV}$ & $\Upsilon(9460)$ & $9461 \mathrm{MeV}$ \\
\hline
\end{tabular}

Table 1. Estimated masses of mesons at confinement scale $\Lambda=220 \mathrm{MeV}$.

\section{QCD Running Coupling in the IR Region}

We consider the meson mass $M$ as an appropriate energy-scale parameter for coupling $\alpha_{s}(M)$. Having adjusted model parameters, we estimate $\alpha_{s}(M)$ in the low-energy domain by exploiting meson masses [ $[\mathbb{8}]$. Then, we perform global evaluation of $\alpha_{s}(M)$ by using formula $\alpha_{s}\left(M_{J}\right)=$ $-1 / \lambda_{J}\left(M_{J}, \Lambda, m_{1}, m_{2}\right)$. The resulting curve at $\Lambda=220 \mathrm{MeV}$ is plotted in Fig. 1 in comparison with recent experimental data [ $[\mathbf{W}]$.

The possibility that the QCD coupling constant features an IR-finite behavior has been extensively studied in recent years (e.g., [12], [13], [4]]). By deriving Eq.([2.3]) for $M=0$ and $m_{1}=m_{2}=0$ we reveal a IR-fixed point

$$
\alpha_{s}^{0}=\alpha_{s}(0)=\frac{3 \pi}{16 \ln (2)} \approx 0.8498 \quad \Rightarrow \quad \alpha_{s}^{0} / \pi=0.2705 .
$$

The obtained IR-fixed value of the coupling constant is moderate and is in a reasonable agreement with often quoted estimates:

$$
\begin{array}{ll}
\alpha_{s}^{0} / \pi \simeq 0.19-0.25 & \text { [प5] }, \\
\alpha_{s}^{0} / \pi \simeq 0.265 & \text { [प6], } \\
\alpha_{s}^{0} / \pi \simeq 0.26 & \text { [ए7] }
\end{array}
$$

and other phenomenological evidences (e.g., [ए]]). 

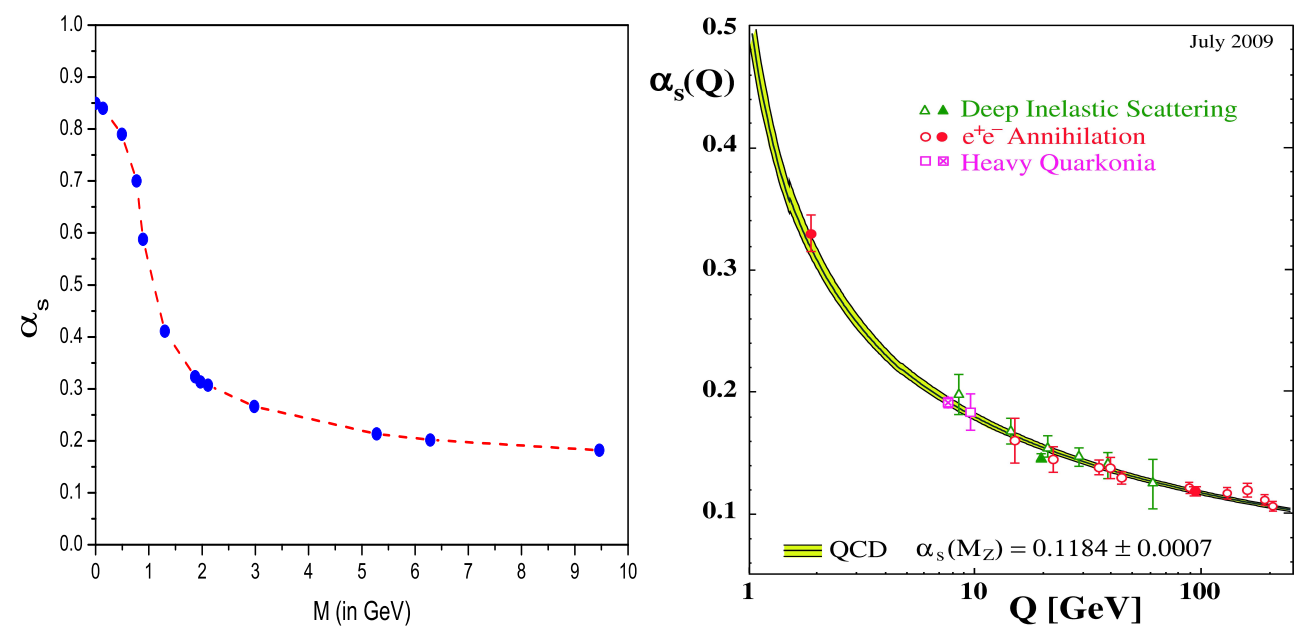

Fig. 1. Our estimate of $\alpha_{s}(M)$ at the confinement scale value $\Lambda=220 \mathrm{MeV}$ (left panel) compared with $\alpha_{s}(Q)$ defined in recent experiments (e.g., [四, []).

\section{Glueball Lowest State}

Because of the confinement, gluons are not observed, they may only come in bound states called glueballs. Glueballs are the most unusual particles predicted by the QCD but not found experimentally yet. There are predictions expecting non- $q \bar{q}$ scalar objects, like glueballs and multiquark states in the mass range $\sim 1500 \div 1800 \mathrm{MeV}$ [마, 며, [20].

The glueball spectrum has been studied by using effective approaches like the QCD sum rules [2]], Coulomb gauge QCD [122], various potential [23] and string models [24] as well as lattice QCD simulations [25, [26].

Below we consider a two-gluon bound states. First, we isolate the color-singlet term in the bi-gluon spin-zero (scalar) and spin-two (tensor) currents. Further we consider only the scalar component. By omitting details of intermediate calculations we define the Bethe-Salpeter kernel for the two-gluon scalar state:

$$
\Pi(z) \doteq \iint d t d s U_{n}(t) \sqrt{W(t)} D\left(\frac{t+s}{2}+z\right) \cdot D\left(\frac{t+s}{2}-z\right) \sqrt{W(s)} U_{n}(s) .
$$

The scalar glueball mass $M_{G}$ is defined from equation:

$$
1-\frac{\pi g^{2}}{8} \int d z e^{i z p} \Pi(z)=0, \quad p^{2}=-M_{G}^{2}
$$

Particularly, for $\Lambda \approx 220 \mathrm{MeV}$ and $\alpha_{s}\left(M_{\tau}\right)=0.343$ we estimate

$$
M_{G} \approx 1792 \mathrm{MeV}
$$

Our estimate is in reasonable agreement with other predictions [ [25, [18, 20], 26]. The quenched lattice estimate favors a scalar glueball mass $M_{G}=1710 \pm 50 \pm 58 \mathrm{MeV}$ [27] while recent glueball mass measurements from improved staggered fermion simulations (UKQCD Collaboration) predict the scalar glueball mass at $M_{G}=1830 \mathrm{MeV}$ [[28]. 
To conclude, the conventional meson spectrum has been estimated within a relativistic quantumfield model based on infrared confinement. We demonstrated that global properties of some lowenergy phenomena may be explained reasonably in the framework of a simple relativistic quantumfield model if one guesses correct symmetry structure of the quark-gluon interaction in the confinement region and uses simple forms of propagators in the hadronization regime. It is shown that the behavior of the QCD running coupling in the low-energy region may be explained reasonably by using the meson spectrum. In doing so, we do not aim to obtain the behavior of the coupling constant at all scales. A new, independent, and specific IR-finite behavior of QCD coupling is exhibited. At moderate mass scale we obtain $\alpha_{s}$ in coincidence with the QCD predictions. However, at large mass scale (above $10 \mathrm{GeV}$ ) $\hat{\alpha}_{s}$ decreases much faster than expected by QCD prediction. The reason is the use of simple confined propagators in the form of entire (exponential) functions. As applications, we performed estimates on conventional meson spectrum and the lowest glueball mass and, the results were in reasonable agreement with experimental data.

\section{References}

[1] K. Nakamura et al., J.Phys. G37 (2010) 075021.

[2] S. Bethke, Eur. Phys. J. C64 (2009) 689 [hep-ph/0908.1135].

[3] D.V. Shirkov, Theor. Math. Phys. 132 (2002) 1309.

[4] O. Kaczmarek and F. Zantow, Phys. Rev. D71 (2005) 114510.

[5] G. Ganbold, Phys. Part. Nucl. 43 (2012) 79.

[6] C. S. Fischer, R. Alkofer and H. Reinhardt, Phys. Rev. D65 (2002) 094008.

[7] C. Lerche and L. von Smekal, Phys. Rev. D65 (2002) 125006.

[8] G. Ganbold, Phys. Rev. D81 (2010) 094008.

[9] G. Ganbold, Phys. Rev. D79 (2009) 034034.

[10] G. Ganbold, J.Phys.: Conf. Ser. 295 (2011) 012041.

[11] M. Baldicchi et al., Phys. Rev. D77 (2008) 034013.

[12] S. J. Brodsky and G. F. de Teramond, Phys. Lett. $\mathbf{B 5 8 2}$ (2004) 211.

[13] A. C. Aguilar, A. Mihara and A. A. Natale, Int. J. Mod. Phys. A19 (2004) 249.

[14] D. V. Shirkov and I. L. Solovtsov, Phys. Rev. Lett. 79 (1997) 1209; D. V. Shirkov, Theor. Math. Phys. 136 (2003) 893 [hep-th/0210013].

[15] S. Godfrey and N. Isgur, Phys. Rev. D32 (1985) 189; T. Barnes, F. E. Close and S. Monaghan, Nucl. Phys. B198 (1983) 380.

[16] T. Zhang and R. Koniuk, Phys. Lett. B261 (1991) 311; C.R. Ji, F. Amiri, Phys. Rev. D42 (1990) 3764.

[17] F. Halzen, G. I. Krein and A. A. Natale, Phys. Rev. D47 (1993) 295.

[18] C. Amsler, N.A. Tornqvist, Phys. Rep. 389 (2004) 61.

[19] D.V. Bugg, Phys. Lett. C397 (2004) 257.

[20] W.-M. Yao et al., J. Phys. G33 (2006) 1. 
[21] S. Narison, Nucl. Phys. B509 (1998) 312; Nucl. Phys. $\mathbf{6 6 7 5}$ (2000) 54.

[22] A.P. Szczepaniak and E.S. Swanson, Phys. Lett. B577 (2003) 61.

[23] A.B. Kaidalov and Yu.A. Simonov, Phys. Lett. B636 (2006) 101.

[24] F. Brau, C. Semay, and B. Silvestre-Brac, Phys. Rev. D70 (2004) 014017.

[25] C.J. Morningstar and M. Peardon, Phys. Rev. D60 (1999) 034509.

[26] H.B. Meyer and M.J. Teper, Phys. Lett. B605 (2005) 344.

[27] Y. Chen et al., Phys. Rev. D73 (2006) 014516.

[28] Christopher M. Richards et al., Phys. Rev. D82 (2010) 034501. 